\title{
Exactly Solvable Schrödinger Equation with Hypergeometric Wavefunctions
}

\author{
J. Morales $^{1 *}$, J. García-Martínez ${ }^{2}$, J. García-Ravelo³ J. J. Peña1 \\ ${ }^{1}$ Universidad Autónoma Metropolitana-Azc., DCB-Area de FAMA, Sn Pablo 180, Mexico City, México \\ ${ }^{2}$ Tecnológico de Estudios Superiores de Ixtapaluca, División de Ingeniería Informática e Ingeniería Biomédica, \\ Carretera Ixtapaluca Coatepec Km. 7, Mexico City, México \\ ${ }^{3}$ Escuela Superior de Física y Matemáticas, IPN-Zacatenco, UP Adolfo López Mateos, México \\ Email: ${ }^{j}$ jmr@correo.azc.uam.mx
}

Received 13 October 2015; accepted 23 November 2015; published 26 November 2015

Copyright (C) 2015 by authors and Scientific Research Publishing Inc.

This work is licensed under the Creative Commons Attribution International License (CC BY). http://creativecommons.org/licenses/by/4.0/

\section{(c) 7 Open Access}

\section{Abstract}

In this work, the canonical transformation method is applied to a general second order differential equation (DE) in order to trasform it into a Schrödinger-like DE. Our proposal is based on an auxiliary function $g(x)$ which determines the transformation needed to find exactly-solvable potentials associated to a known DE. To show the usefulness of the proposed approach, we consider explicitly their application to the hypergeometric DE with the aim to find quantum potentials with hypergeometric wavefunctions. As a result, different potentials are obtained depending on the choice of the auxiliary function; the generalized Scarf, Posh-Teller, Eckart and Rosen-Morse trigonometric and hyperbolic potentials, are derived by selecting $g(x)$ as constant and proportional to the $P(x)$ hypergeometric coefficient. Similarly, the choices $g(x) \sim P(x) / x^{2}$ and $g(x) \sim x^{2} / P(x)$ give rise to a class of exactly-solvable generalized multiparameter exponential-type potentials, which contain as particular cases the Hulthén, Manning-Rosen and Woods-Saxon models, among others. Our proposition is general and can be used with other important DE within the frame of applied matematics and physics.

\section{Keywords}

Canonical Transformation, Schrödinger-Like Equation, Hypergeometric DE, Exactly-Solvable Potentials

\section{Introduction}

Exactly and quasi-exactly solvable potential models are important in practically any field of theoretical quantum

${ }^{*}$ Corresponding author.

How to cite this paper: Morales, J., García-Martínez, J., García-Ravelo, J. and Peña, J.J. (2015) Exactly Solvable Schrödinger Equation with Hypergeometric Wavefunctions. Journal of Applied Mathematics and Physics, 3, 1454-1471. 
chemistry and physics for two principal reasons: first, they are useful to understand the behavior of quantum systems and second, can be used as a basis to study problems that can only be treated using perturbative and nonperturbative procedures. In spite of the above, the exactly solvable Schrödinger equations are rather scanty and in their research different analytical or operational approaches have been used. Also, the well known exactly- solvable Scarf, Eckart, Rosen-Morse I and II, Poschl-Teller I and II as well as Hulthén, Manning-Rosen and Woods-Saxon potentials, all they have, as common feature, hypergeometric wavefunctions. Similarly, the harmonic oscillator, Morse, Coulomb or Kratzer potential models have confluent hypergeometric solutions. Consequently, it becomes clear that the exact solution for the Schrödinger equation is reduced to the study of hypergeometric and/or confluent hypergeometric Differential Equations (DE). At this regard, many efforts have been conducted to find the intermapping between different solvable potentials [1] [2] with the aim to give a unified treatment of partner potentials [3]. For example, in the case of potentials with the hypergeometric wavefunctions, the hexagonal diagram proposed by Cooper et al. [4] is very useful to show how all the shape invariant potentials are inter-related. Also, it has been proposed a pre-potential approach to study of Eckart-type potentials [5] and a five-parameter exponential-type potential to unify the treatment of exactly solvable trigonometric potential models [6]-[8]. Furthermore, to find exactly solvable Schrödinger equations different methods based on Supersymetric Quantum Mechanics (SUSY-QM) [9], Asymptotic Iteration Method (AIM) [10] and on the transformation of a Schrödinger equation into a hypergeometric-type DE by the Nikiforov-Uvarov (NU) [11] approach, have been used. In this work, we present a proposal that can be considered inverse to the NU method. However, instead of transforming a DE into a Schrödinger equation we consider the transformation of a general homogeneous linear second order DE to their canonical form. Obviously, the general DE has as particular cases the hypergeometric and confluent hypergeometric DE. For that reason, this work aims at finding solvable potentials with hypergeometric wavefunctions leaving the treatment of models with confluent hypergeometric solutions elsewhere [12]. Thus, the proposed approach to transform a general DE into a Schrödinger-like equation is given in next section by means of the canonical transformation method given in the Appendix. The application of the present proposal is given in Section 3, where we consider the hypergeometric DE by means of an auxiliary function $g(x)$, defined in Section 2, that indicates the required transformation. That is, as will be shown, each possibility of $g(x)$ leads to different generalized potentials which are reduced to well known particular cases. Finally, in Section 4 we presents the concluding remarks emphasizing that our proposition is general for which can be directly applied to other important DE.

\section{Transformation of a General DE into a Schrödinger-Like Equation}

According to the proposition given in Equation (A5), the generalized canonical transformation of Equation (A1) becomes

$$
\frac{P(F(r))}{\left(F^{\prime}(r)\right)^{2}} \frac{\mathrm{d}^{2} f(F(r))}{\mathrm{d} r^{2}}+\left(\frac{Q(F(r))}{F^{\prime}(r)}-\frac{P(F(r)) F^{\prime \prime}(r)}{\left(F^{\prime}(r)\right)^{3}}\right) \frac{\mathrm{d} f(F(r))}{\mathrm{d} r}+R(F(r)) f(F(r))=0,
$$

that can be rewritten as

$$
\frac{\mathrm{d}^{2} f(F(r))}{\mathrm{d} r^{2}}+2 W(r) \frac{\mathrm{d} f(F(r))}{\mathrm{d} r}+g(F(r)) R(F(r)) f(F(r))=0,
$$

where

$$
W(r)=\frac{g(F(r)) Q(F(r))-F^{\prime \prime}(r)}{2 F^{\prime}(r)}
$$

and

$$
g(F(r))=\frac{\left(F^{\prime}(r)\right)^{2}}{P(F(r))}
$$

that will be referred as auxiliary function hereafter. Consequently, from the above, the variable $r$ is given by 


$$
r= \pm \int^{F(r)} \frac{\mathrm{d} t}{\sqrt{g(t) P(t)}}=\phi(F(r))
$$

in such a way that Equation (A5) is feasible on condition to have the inverse function

$$
F(r)=\phi^{-1}(r) \text {. }
$$

Furthermore, the general transform given in Equation (A6) can be rewritten as

$$
f(F(r))=\psi(r) \exp \left[-\int^{r} W(t) \mathrm{d} t\right]
$$

where we have used the auxiliary function and the fact that

$$
\sqrt{F^{\prime}(r)}=\exp \left(\frac{1}{2} \ln F^{\prime}(r)\right)=\exp \left(\frac{1}{2} \int \frac{\mathrm{d}}{\mathrm{d} r} \ln F^{\prime}(r) \mathrm{d} r\right)=\exp \left(\frac{1}{2} \int \frac{F^{\prime \prime}(r)}{F^{\prime}(r)} \mathrm{d} r\right),
$$

such that

$$
\sqrt{F^{\prime}(r)} \exp \left(-\frac{1}{2} \int \frac{Q(F(r))}{P(F(r))} \mathrm{d} F(r)\right)=\exp \left(-\frac{1}{2} \int\left(\frac{Q(F(r)) \mathrm{d} F(r)}{P(F(r)) \mathrm{d} r}-\frac{F^{\prime \prime}(r)}{F^{\prime}(r)}\right) \mathrm{d} r\right) .
$$

Consequently, by substituting Equation (7) into Equation (2), the generalized canonical form of Equation (A1) will be

$$
-\frac{\mathrm{d}^{2} \psi(r)}{\mathrm{d} r^{2}}+\left(W^{2}(r)+W^{\prime}(r)-g(F(r)) R(F(r))\right) \psi(r)=0 .
$$

At this point, it should be noticed that above equation can be identified with a Schrödinger-like equation where the $\psi(r)$ wavefunction can be obtained from Equation (7) on condition that coefficients $P(x), Q(x), R(x)$ and the solution $f(x)$ of Equation (A1) are known. Besides, the potential and energy spectra are given in general by

$$
W^{2}(r)+W^{\prime}(r)-g(F(r)) R(F(r))=V\left(r, a_{i}, b_{j}\right)-E\left(a_{i}, b_{j}\right),
$$

being $\left\{a_{i}\right\}$ and $\left\{b_{j}\right\}$ a set of parameters that come from Equation (A1) and Equation (5) respectively.

In short, it is worth noting that $x=F(r)$ is involved in the construction of potential $V\left(r, a_{i}, b_{j}\right)$ as well as in its corresponding solutions $\psi(r)$ given by Equation (7) through the function $W(r)$. Also, according to Equation (4), there will be different transformations $F(r)$ depending on the selection of the auxiliary function $g(F(r))$ which means the existence of various exactly-solvable potentials having the same wavefunctions of the former DE.

\section{Application to the Hypergeometric DE}

To show the usefulness of the approach given in above section, in the search of exactly-solvable Schrödinger equations, let us apply the above results to the hypergeometric DE

$$
x(1-x) y^{\prime \prime}(x)+[c-(a+b+1) x] y^{\prime}(x)-a b y(x)=0
$$

whose solution is [13]

$$
y(x)={ }_{2} F_{1}(a, b ; c ; x)=\sum_{\kappa=0}^{\infty} \frac{(a)_{\kappa}(b)_{\kappa}}{(c)_{\kappa}} \frac{x^{\kappa}}{\kappa !} .
$$

where $(t)_{n}=(t)(t+1)(t+2) \cdots(t+n-1),(t)_{0}=1$ is the Pochhammer symbol and parameters $a$ and $b$ are constant with $c \neq 0,-1,-2,-3, \cdots$. That is, in this case Equation (12) matches with Equation (A1) provided that $f(x)=y(x), P(x)=x(1-x), Q(x)=c-(a+b+1) x, R(x)=-a b$ and $\left\{a_{i}\right\}=\{a, b, c\}$.

As mentioned before, depending on the choice of $F(r)$, or their corresponding auxiliary function $g(F(r))$, 
our proposal leads to different exactly-solvable potentials. Consequently, the different options for $g(F(r))$ can be obtained by Equation (3) given by

$$
W(r)=c \frac{g(F(r))}{2 F^{\prime}(r)}-(a+b+1) \frac{g(F(r)) F(r)}{2 F^{\prime}(r)}-\frac{1}{2} \frac{F^{\prime \prime}(r)}{F^{\prime}(r)},
$$

where we have used the explicit form of function $Q(x)$. So, in order to have at least one constant term associated to the eigenvalue $E\left(a_{i}, b_{j}\right)$ of Equation (11), one option is to propose the following cases:

$c \frac{g(F(r))}{2 F^{\prime}(r)}=$ const, $(a+b+1) \frac{g(F(r)) F(r)}{2 F^{\prime}(r)}=$ const and $\frac{1}{2} \frac{F^{\prime \prime}(r)}{F^{\prime}(r)}=$ const. So, by using Equation (4), the first option leads to $g(F(r)) \sim P(x)$, while the second implies $g(F(r)) \sim P(x) / x^{2}$ and the third option gives $g(F(r)) \sim x^{2} / P(x)$. Another possibility arises when $g(F(r)) R(F(r))=$ const, which means $g(F(r))=$ const since $R(x)=-a b$. All these options are considered explicitly in what follows by using $g(x)$ instead of $g(F(r))$.

\subsection{Generalized Potentials from $g(x)$ Constant}

Let us consider the identity

$$
P(x)=\frac{1}{4}\left[1-(1-2 x)^{2}\right]
$$

to rewrite Equation (5) as

$$
r= \pm \int^{F(r)} \frac{2 \mathrm{~d} t}{\sqrt{g(t)\left[1-(1-2 t)^{2}\right]}}
$$

with the purpose to use the integral [14]

$$
\int \frac{\mathrm{d} w}{\sqrt{m^{2} \pm w^{2}}}=i^{1 / 2 \mp 1 / 2} \sinh ^{-1}\left( \pm i^{1 / 2 \mp 1 / 2}\left(\frac{w}{m}\right)\right) .
$$

That is, the above integral and the choice of $g(x)$ as constant given by

$$
g(x)= \pm \frac{1}{4} k^{2},
$$

allows to evaluate the integral of Equation (16) as

$$
r= \pm \frac{4}{k} \int^{F(r)} \frac{\mathrm{d} t}{\sqrt{\left(i^{1 / 2 \mp 1 / 2}\right)^{2} \mp(1-2 t)^{2}}}= \pm\left(-\frac{2}{k}\right) i^{1 / 2 \pm 1 / 2} \sinh ^{-1}(i(1-2 F(r))) .
$$

Consequently, in this case the corresponding transformation is

$$
F^{ \pm}(r)=\frac{1}{2}\left(1 \pm i \sinh \left(i^{-1 / 2 \pm 1 / 2} \frac{k r}{2}\right)\right),
$$

leading to the particular cases + and - specified by

$$
F^{ \pm}(r)= \begin{cases}\frac{1}{2}\left(1+\sin \left(\frac{k r}{2}\right)\right) & -\pi / k<r<\pi / k \\ \frac{1}{2}\left(1-i \sinh \left(\frac{k r}{2}\right)\right) & -\infty<r<\infty .\end{cases}
$$

Thus, according to Equation (3), $W^{ \pm}(r)$ becomes 


$$
W^{ \pm}(r)=\frac{k}{4} i^{-1 / 2 \pm 1 / 2}\left((2 c-(a+b+1)) \operatorname{sech}\left(i^{-1 / 2 \mp 1 / 2} \frac{k r}{2}\right) \mp i(a+b) \tanh \left(i^{-1 / 2 \mp 1 / 2} \frac{k r}{2}\right)\right)
$$

which means

$$
W^{+}(r)=\frac{k}{4}\left(i(a+b+1-2 c) \operatorname{sech}\left(\frac{k r}{2}\right)+(a+b) \tanh \left(\frac{k r}{2}\right)\right)
$$

and

$$
W^{-}(r)=-\frac{k}{4}\left((a+b+1-2 c) \sec \left(\frac{k r}{2}\right)+(a+b) \tan \left(\frac{k r}{2}\right)\right) .
$$

Thus, as mentioned before, the potential function $V(r)=V\left(r, a_{i}, b_{j}\right)$ and the eigenvalue $E=E\left(a_{i}, b_{j}\right)$ can be identified from Equation (11) as

$$
\begin{aligned}
& \left(W^{ \pm}(r)\right)^{2}+\left(W^{ \pm}(r)\right)^{\prime}-g(F(r)) R(F(r)) \\
& =\frac{k^{2}}{16}\left[ \pm(a+b+1-2 c)^{2}+(a+b)(a+b-2) \operatorname{sech}^{2}\left(i^{-1 / 2 \mp 1 / 2} \frac{k r}{2}\right)\right. \\
& \left.+2 i(a+b+1-2 c)(a+b-1) \operatorname{sech}\left(i^{-1 / 2 \mp 1 / 2} \frac{k r}{x}\right) \tanh \left(i^{-1 / 2 \mp 1 / 2} \frac{k r}{2}\right) \mp(a+b)^{2} \pm 4 a b\right]
\end{aligned}
$$

leading to the corresponding generalized potentials $V^{ \pm}(r)$

$$
\begin{aligned}
V^{ \pm}(r)= & \frac{k^{2}}{16}\left[ \pm\left((2 c-(a+b+1))^{2}+(a+b)(a+b-2)\right) \operatorname{sech}^{2}\left(i^{-1 / 2 \mp 1 / 2} \frac{k r}{2}\right)\right. \\
& \left.+2 i(2 c-(a+b+1))(1-(a+b)) \operatorname{sech}\left(i^{-1 / 2 \mp 1 / 2} \frac{k r}{2}\right) \tanh \left(i^{-1 / 2 \mp 1 / 2} \frac{k r}{2}\right)\right]
\end{aligned}
$$

with eigenvalues

$$
E^{ \pm}= \pm \frac{k^{2}}{16}\left((a+b)^{2}-4 a b\right)
$$

and eigenfunctions

$$
\begin{aligned}
\psi^{ \pm}(r)= & \exp \left( \pm \frac{i}{2}(2 c-(a+b+1)) \tan ^{-1}\left(\sinh \left(i^{-1 / 2 \mp 1 / 2} \frac{k r}{2}\right)\right)\right) \\
& \times \cosh ^{\frac{a+b}{2}}\left(i^{-1 / 2 \mp 1 / 2} \frac{k r}{2}\right){ }_{2} F_{1}\left(a, b ; c ; \frac{1 \pm i \sinh \left(i^{-1 / 2 \mp 1 / 2} \frac{k r}{2}\right)}{2}\right) .
\end{aligned}
$$

Also, in order to have physically acceptable wavefunctions, the original parameters $a, b$ and $c$ are redefined as

$$
a=-n, \quad b=n+s, \quad c=\frac{1}{2}\left(i^{-1 / 2 \pm 1 / 2} q+s+1\right),
$$

with $n=0,1,2,3, \cdots$, for which the potentials of Equation (26) become

$$
\begin{aligned}
V^{ \pm}(r)= & \frac{k^{2}}{16}\left[\left(q^{2} \pm s(s-2)\right) \operatorname{sech}^{2}\left(i^{-1 / 2 \mp 1 / 2} \frac{k r}{2}\right)\right. \\
& \left.+2 i^{1 / 2 \pm 1 / 2} q(1-s) \operatorname{sech}\left(i^{-1 / 2 \mp 1 / 2} \frac{k r}{2}\right) \tanh \left(i^{-1 / 2 \mp 1 / 2} \frac{k r}{2}\right)\right]
\end{aligned}
$$

as well as their respective wavefunctions 


$$
\begin{aligned}
\psi_{n}^{ \pm}(r)= & \cosh ^{s / 2}\left(i^{-1 / 2 \mp 1 / 2} \frac{k r}{2}\right) \exp \left( \pm i^{1 / 2 \pm 1 / 2} \frac{q}{2} \tan ^{-1}\left(\sinh \left(i^{-1 / 2 \mp 1 / 2} \frac{k r}{2}\right)\right)\right) \\
& \times{ }_{2} F_{1}\left(-n, n+s ; \frac{s+1+i^{-1 / 2 \pm 1 / 2} q}{2} ; \frac{1 \pm i \sinh \left(i^{-1 / 2 \mp 1 / 2} \frac{k r}{2}\right)}{2}\right)
\end{aligned}
$$

with energy spectra

$$
E_{n}^{ \pm}= \pm \frac{k^{2}}{16}(2 n+s)^{2}
$$

At this point, is important to notice that particular cases

$$
V^{+}(r)=\frac{k^{2}}{16}\left(\left(q^{2}+s(s-2)\right) \sec ^{2}\left(\frac{k r}{2}\right)+2 q(1-s) \sec \left(\frac{k r}{2}\right) \tan \left(\frac{k r}{2}\right)\right)
$$

and

$$
V^{-}(r)=\frac{k^{2}}{16}\left(\left(q^{2}-s(s-2)\right) \operatorname{sech}^{2}\left(\frac{k r}{2}\right)+2 q(1-s) \operatorname{sech}\left(\frac{k r}{2}\right) \tanh \left(\frac{k r}{2}\right)\right)
$$

are identified with the well known trigonometric $\left(V^{+}(r)\right)$ and hyperbolic $\left(V^{-}(r)\right)$ Scarf potentials having as eigenfunctions

$$
\psi_{n}^{+}(r)=\cos ^{s / 2}\left(\frac{k r}{2}\right)\left(\sec \left(\frac{k r}{2}\right)+\tan \left(\frac{k r}{2}\right)\right)^{\frac{q}{2}} P_{n}^{\frac{s+q-1}{2}, \frac{s-q-1}{2}}\left(\sin \left(\frac{k r}{2}\right)\right)
$$

and

$$
\psi_{n}^{-}(r)=\cosh ^{s / 2}\left(\frac{k r}{2}\right) \exp \left(-\frac{q}{2} \tan ^{-1}\left(\sinh \left(\frac{k r}{2}\right)\right)\right) P_{n}^{\frac{s-i q-1}{2}, \frac{s+i q+1}{2}}\left(i \sinh \left(\frac{k r}{2}\right)\right)
$$

respectively, where ${ }_{2} F_{1}(-n, \alpha+\beta+n+1 ; \alpha+1 ;(1-x) / 2)$ is proportional to the $P_{n}^{\alpha, \beta}(x)$ Jacobi polynomials. As a consequence the following question arises: From where comes the Posch-Teller potential? The answer to that question follows from the negative of $g(x)$ given in Equation (18) but now along with the integral [14]

$$
\int \frac{\mathrm{d} w}{\sqrt{w^{2} \mp m^{2}}}=\left\{\begin{array}{l}
\cosh ^{-1}\left(\frac{w}{m}\right) \\
\sinh ^{-1}\left(\frac{w}{m}\right)
\end{array}\right\} .
$$

As can be proved, the plus $(+)$ case leads to $V^{+}(r)$ given in Equation (33). So, we are going to consider only the minus $(-)$ case. That is, now Equation (16) gives rise to

$$
r=\frac{2}{k} \cosh ^{-1}(1-2 F),
$$

which indicates that the corresponding transformation is

$$
F(r)=\frac{1}{2}\left(1-\cosh \left(\frac{k r}{2}\right)\right)
$$

Then, according to Equation (3), in this case $W(r)$ will be

$$
W(r)=\frac{k}{4}\left(-(a+b+1-2 c) \operatorname{csch}\left(\frac{k r}{2}\right)+(a+b) \operatorname{coth}\left(\frac{k r}{2}\right)\right)
$$

leading, from Equation (11), to the potential 


$$
\begin{aligned}
V(r)= & \frac{k^{2}}{16}\left[\left((a+b+1-2 c)^{2}+(a+b)(a+b-2)\right)\right] \operatorname{csch}^{2}\left(\frac{k r}{2}\right) \\
& \left.-2(a+b-1)(a+b+1-2 c) \operatorname{csch}\left(\frac{k r}{2}\right) \operatorname{coth}\left(\frac{k r}{2}\right)\right],
\end{aligned}
$$

with eigenvalue

$$
E=-\frac{k^{2}}{16}(a-b)^{2}
$$

and eigenfunction

$$
\psi(r)=\left(\tanh \left(\frac{k r}{4}\right)\right)^{-\frac{a+b+1-2 c}{2}}\left(\sinh \left(\frac{k r}{2}\right)\right)^{\frac{a+b}{2}}{ }_{2} F_{1}\left(a, b ; c ; \frac{1-\cosh \left(\frac{k r}{2}\right)}{2}\right) .
$$

Finally, using the conditions for physically acceptable wavefunctions one obtains

$$
a=-n, \quad b=n+s, \quad c=\frac{1}{2}(1+s-q) .
$$

that leads to the potential

$$
V(r)=\frac{k^{2}}{16}\left[\left(q^{2}+s^{2}-2 s\right) \operatorname{csch}^{2}\left(\frac{k r}{2}\right)-2 q(s-1) \operatorname{csch}\left(\frac{k r}{2}\right) \operatorname{coth}\left(\frac{k r}{2}\right)\right],
$$

with eigenfunctions

$$
\psi_{n}(r)=\left(\tanh \left(\frac{k r}{4}\right)\right)^{-q / 2}\left(\sinh \left(\frac{k r}{2}\right)\right)^{s / 2} P_{n}^{\left(\frac{s-q-1}{2}, \frac{s+q-1}{2}\right)}\left(\cosh \left(\frac{k r}{2}\right)\right)
$$

and energy spectra

$$
E_{n}=-\frac{k^{2}}{16}(2 n+s)^{2}
$$

that coincides with the well known Posch-Teller potential.

\subsection{Generalized $V(r)$ from $g(x)$ Proportional to $P(x)$}

Similarly to the cases considered in above section, the choice of

$$
g(x)=\frac{k^{2}}{4}\left[1-(1-2 x)^{2}\right]=k^{2} P(x), \quad k>0,
$$

can be worked by means of the integrals

$$
\int \frac{\mathrm{d} w}{m^{2}-w^{2}}=\left\{\begin{array}{ll}
\frac{1}{m} \tanh ^{-1}\left(\frac{w}{m}\right) & w^{2}<m^{2} \\
\frac{1}{m} \operatorname{coth}^{-1}\left(\frac{w}{m}\right) & w^{2}>m^{2}
\end{array} .\right.
$$

In fact, the use of Equation (49) lets write Equation (16) as

$$
r= \pm \frac{2}{k} \begin{cases}\tanh ^{-1}(1-2 F(r)), & (1-2 F(r))^{2}<1 \\ \operatorname{coth}^{-1}(1-2 F(r)), & (1-2 F(r))^{2}>1\end{cases}
$$

leading to the corresponding transformation 


$$
F^{ \pm}(r)=\frac{1}{2}\left(1-\tanh \left(\frac{i \pi}{2}\left(\frac{1}{2} \mp \frac{1}{2}\right)+\frac{k r}{2}\right)\right)=\frac{1}{2}\left\{\begin{array}{cc}
1-\tanh \left(\frac{k r}{2}\right), & -\infty<r<\infty \\
1-\operatorname{coth}\left(\frac{k r}{2}\right), & r>0
\end{array}\right\} .
$$

So, according to Equation (3)

$$
W^{ \pm}(r)=\frac{k}{4}\left[(a+b+1-2 c)-(a+b-1) \tanh \left(\frac{i \pi}{2}\left(\frac{1}{2} \mp \frac{1}{2}\right)+\frac{k r}{2}\right)\right] .
$$

Thus, the potential function $V(r)$ and the eigenvalue $E$ can be identified from Equation (11) as

$$
\begin{aligned}
& \left(W^{ \pm}(r)\right)^{2}+\left(W^{ \pm}(r)\right)^{\prime}-g\left(F^{ \pm}(r)\right) R\left(F^{ \pm}(r)\right) \\
& =-\frac{k^{2}}{16}\left[2(a+b+1-2 c)(a+b-1) \tanh \left(\frac{i \pi}{2}\left(\frac{1}{2} \mp \frac{1}{2}\right)+\frac{k r}{2}\right)\right. \\
& \left.+\left((a-b)^{2}-1\right) \operatorname{sech}^{2}\left(\frac{i \pi}{2}\left(\frac{1}{2} \mp \frac{1}{2}\right)+\frac{k r}{2}\right)-\left((a+b+1-2 c)^{2}+(a+b-1)^{2}\right)\right]
\end{aligned}
$$

to obtain the corresponding generalized potentials

$$
\begin{aligned}
V^{ \pm}(r)= & -\frac{k^{2}}{16}\left[2(a+b+1-2 c)(a+b-1) \tanh \left(\frac{i \pi}{2}\left(\frac{1}{2} \mp \frac{1}{2}\right)+\frac{k r}{2}\right)\right. \\
& \left.+\left((a-b)^{2}-1\right) \operatorname{sech}^{2}\left(\frac{i \pi}{2}\left(\frac{1}{2} \mp \frac{1}{2}\right)+\frac{k r}{2}\right)\right],
\end{aligned}
$$

with eigenvalue

$$
E=-\frac{k^{2}}{16}\left((a+b+1-2 c)^{2}+(a+b-1)^{2}\right)
$$

and wavefunction

$$
\begin{aligned}
\psi^{ \pm}(r)= & \exp \left(\frac{k}{4}(a+b+1-2 c) r\right) \cosh ^{(a+b-1) / 2}\left(\frac{i \pi}{2}\left(\frac{1}{2} \mp \frac{1}{2}\right)+\frac{k r}{2}\right) \\
& \times{ }_{2} F_{1}\left(a, b, c ; \frac{1}{2}\left(1-\tanh \left(\frac{i \pi}{2}\left(\frac{1}{2} \mp \frac{1}{2}\right)+\frac{k r}{2}\right)\right)\right) .
\end{aligned}
$$

As before, with the aim to have physically acceptable solutions it becomes necessary to redefine the original parameters $a, b$ and $c$ as follow

$$
a=b=-n, \quad c=-\frac{4 n^{2}-p-1}{2(2 n+1)},
$$

with $n=0,1,2,3, \cdots$ and $p>0$. This fact, leads to

$$
V^{ \pm}(r)=-\frac{k^{2}}{16}\left(2 p \tanh \left(\frac{i \pi}{2}\left(\frac{1}{2} \mp \frac{1}{2}\right)+\frac{k r}{2}\right)-\operatorname{sech}^{2}\left(\frac{i \pi}{2}\left(\frac{1}{2} \mp \frac{1}{2}\right)+\frac{k r}{2}\right)\right),
$$

with their corresponding wavefunctions

$$
\psi_{n}^{ \pm}(r)=\exp \left(\frac{-k p r}{4(2 n+1)}\right)\left(\cosh \left(\left(\frac{i \pi}{2}\left(\frac{1}{2} \mp \frac{1}{2}\right)+\frac{k r}{2}\right)\right)\right)^{n+1 / 2} P_{n}^{(\alpha, \beta)}\left(\tanh \left(\frac{i \pi}{2}\left(\frac{1}{2} \mp \frac{1}{2}\right)+\frac{k r}{2}\right)\right),
$$

and energy spectra 


$$
E_{n}^{ \pm}=-\frac{k^{2}}{16}\left((2 n+1)^{2}+\frac{p^{2}}{(2 n+1)^{2}}\right)
$$

where

$$
\alpha=-\frac{(2 n+1)^{2}-p}{2(2 n+1)} \text { and } \beta=-\frac{(2 n+1)^{2}+p}{2(2 n+1)} .
$$

At this point, is important to notice that potential $V^{+}(r)$ given by

$$
V^{+}(r)=-\frac{k^{2}}{16}\left(2 p \tanh \left(\frac{k r}{2}\right)-\operatorname{sech}^{2}\left(\frac{k r}{2}\right)\right),
$$

corresponds to the Rosen-Morse II hyperbolic potential and

$$
V^{-}(r)=-\frac{k^{2}}{16}\left(2 p \operatorname{coth}\left(\frac{k r}{2}\right)+\operatorname{csch}^{2}\left(\frac{k r}{2}\right)\right)
$$

to the exactly solvable Eckart potential. Also, we want to point out that $V^{+}(r)$ and $V^{-}(r)$ are isospectral, accordingly to Equation (60), with wavefunctions

$$
\psi_{n}^{+}(r)=\exp \left(\frac{-k p r}{4(2 n+1)}\right) \cosh ^{n+1 / 2}\left(\frac{k r}{2}\right) P_{n}^{(\alpha, \beta)}\left(\tanh \left(\frac{k r}{2}\right)\right),
$$

and

$$
\psi_{n}^{-}(r)=\exp \left(\frac{-k p r}{4(2 n+1)}\right) \sinh ^{n+1 / 2}\left(\frac{k r}{2}\right) P_{n}^{(\alpha, \beta)}\left(\operatorname{coth}\left(\frac{k r}{2}\right)\right),
$$

respectively. Similarly to the cases analyzed in section III.1), the question is now; How can obtain the Rosen-Morse II trigonometric potential? To answer that question, we are going to use the same choice of $g(x)$ but now proportional to the negative $P(x)$. That is,

$$
g(x)=-k^{2} P(x)
$$

that together with Equation (49) in Equation (16) permit us to obtain

$$
r= \pm \frac{2 i}{k}\left\{\begin{array}{ll}
\tanh ^{-1}(1-2 F(r)), & (1-2 F(r))^{2}<1 \\
\operatorname{coth}^{-1}(1-2 F(r)), & (1-2 F(r))^{2}>1
\end{array}\right\} .
$$

Thus, with the aid of the identity

$$
\tan \left(i\left(x+\frac{\pi}{2}\left(\frac{1}{2} \mp \frac{1}{2}\right)\right)\right)=\left\{\begin{array}{c}
i \tan x \\
-i \cot x
\end{array}\right\}
$$

the corresponding transformation is

$$
F^{ \pm}(r)=\frac{1}{2}\left(1 \mp i \tan \left(\frac{\pi}{2}\left(\frac{1}{2} \mp \frac{1}{2}\right)+\frac{k r}{2}\right)\right)=\frac{1}{2}\left\{\begin{array}{lc}
1-i \tan \left(\frac{k r}{2}\right), & -\frac{\pi}{k}<r<\frac{\pi}{k} \\
1-i \cot \left(\frac{k r}{2}\right), & 0<r<\frac{2 \pi}{k}
\end{array}\right\} .
$$

Consequently, according to Equation (3)

$$
W^{ \pm}(r)=\frac{k}{4}\left[ \pm i(a+b+1-2 c)+(a+b-1) \tan \left(\frac{\pi}{2}\left(\frac{1}{2} \mp \frac{1}{2}\right)+\frac{k r}{2}\right)\right],
$$

such that 


$$
\begin{aligned}
& \left(W^{ \pm}(r)\right)^{2}+\left(W^{ \pm}(r)\right)^{\prime}-g\left(F^{ \pm}(r)\right) R\left(F^{ \pm}(r)\right) \\
& =\frac{k^{2}}{16}\left[-\left((a+b+1-2 c)^{2}+(a+b-1)^{2}\right)+\left((a-b)^{2}-1\right) \sec ^{2}\left(\frac{\pi}{2}\left(\frac{1}{2} \mp \frac{1}{2}\right)+\frac{k r}{2}\right)\right. \\
& \left.\quad \pm 2 i(a+b+1-2 c)(a+b-1) \tan \left(\frac{\pi}{2}\left(\frac{1}{2} \mp \frac{1}{2}\right)+\frac{k r}{2}\right)\right],
\end{aligned}
$$

leading to

$$
\begin{aligned}
V^{ \pm}(r)= & \frac{k^{2}}{16}\left[\left((a-b)^{2}-1\right) \sec ^{2}\left(\frac{\pi}{2}\left(\frac{1}{2} \mp \frac{1}{2}\right)+\frac{k r}{2}\right)\right. \\
& \left. \pm 2 i(a+b+1-2 c)(a+b-1) \tan \left(\frac{\pi}{2}\left(\frac{1}{2} \mp \frac{1}{2}\right)+\frac{k r}{2}\right)\right]
\end{aligned}
$$

with eigenvalue

$$
E=\frac{k^{2}}{16}\left((a+b+1-2 c)^{2}+(a+b-1)^{2}\right)
$$

and eigenfunction

$$
\begin{aligned}
\psi^{ \pm}(r)= & \left.\exp \left( \pm \frac{i k}{4}(a+b+1-2 c) r\right)\left(\cos \left(-\frac{\pi}{2}\left(\frac{1}{2} \mp \frac{1}{2}\right)+\frac{k r}{2}\right)\right)\right)^{-(a+b-1) / 2} \\
& \times{ }_{2} F_{1}\left(a, b, c ; \frac{1}{2}\left(1 \mp i \tan \left(\frac{\pi}{2}\left(\frac{1}{2} \mp \frac{1}{2}\right)+\frac{k r}{2}\right)\right)\right) .
\end{aligned}
$$

Similarly, to have physically acceptable wavefunctions the original parameters $a, b$ and $c$ are defined as

$$
a=b=-n, \quad c=-\frac{4 n^{2}-1+i p}{2(2 n+1)},
$$

where $n=0,1,2,3, \cdots$ and $p$ a new real parameter. Consequently, the potentials are corrected as

$$
V^{ \pm}(r)=\frac{k^{2}}{16}\left[-\sec ^{2}\left(\frac{\pi}{2}\left(\frac{1}{2} \mp \frac{1}{2}\right)+\frac{k r}{2}\right) \pm 2 p \tan \left(\frac{\pi}{2}\left(\frac{1}{2} \mp \frac{1}{2}\right)+\frac{k r}{2}\right)\right],
$$

with energy spectra

$$
E_{n}^{ \pm}=\frac{k^{2}}{16}\left((2 n+1)^{2}-\frac{p^{2}}{(2 n+1)^{2}}\right)
$$

and eigenfunctions

$$
\psi_{n}^{ \pm}(r)=\exp \left( \pm \frac{k p r}{4(2 n+1)}\right)\left(\cos \left(-\frac{\pi}{2}\left(\frac{1}{2} \mp \frac{1}{2}\right)+\frac{k r}{2}\right)\right)^{n+1 / 2} P_{n}^{(\alpha, \beta)}\left(\mp i \tan \left(\frac{\pi}{2}\left(\frac{1}{2} \mp \frac{1}{2}\right)+\frac{k r}{2}\right)\right)
$$

where

$$
\alpha=-\frac{(2 n+1)^{2}+i p}{2(2 n+1)} \text { and } \beta=-\frac{(2 n+1)^{2}-i p}{2(2 n+1)} .
$$

Specifically, the $V^{+}(r)$ and $V^{-}(r)$ are given by

$$
V^{+}(r)=\frac{k^{2}}{16}\left(2 p \tan \left(\frac{k r}{2}\right)-\sec ^{2}\left(\frac{k r}{2}\right)\right)
$$


and

$$
V^{-}(r)=\frac{k^{2}}{16}\left(2 p \cot \left(\frac{k r}{2}\right)-\csc ^{2}\left(\frac{k r}{2}\right)\right)
$$

with their respective wavefunctions

$$
\psi_{n}^{+}(r)=\exp \left(-\frac{k p r}{4(2 n+1)}\right)\left(\cos \left(\frac{k r}{2}\right)\right)^{n+1 / 2} P_{n}^{(\alpha, \beta)}\left(i \tan \left(\frac{k r}{2}\right)\right)
$$

and

$$
\psi_{n}^{-}(r)=\exp \left(-\frac{k p r}{4(2 n+1)}\right)\left(\sin \left(\frac{k r}{2}\right)\right)^{n+1 / 2} P_{n}^{(\alpha, \beta)}\left(i \cot \left(\frac{k r}{2}\right)\right)
$$

with the same energy spectra. At this point, we want to notice that potential $V^{-}(r)$ corresponds, as expected, to the exactly solvable Rosen-Morse II trigonometric potential and $V^{+}(r)$ seems to be a new exactly solvable potential.

\subsection{A class of Multiparameter Exponential-Type Potential}

a). Option with $g(x) \sim \frac{x^{2}}{P(x)}$.

According to the third option for $g(x)$ in this case one have

$$
g(x)=\frac{x}{k^{2}(1-x)} \quad k>0 \text { and } x \neq 1 .
$$

In fact, by choosing the minus sign in Equation (5) and the down integral limit as a new parameter $q$, one obtains

$$
r=-\int_{q}^{F(r)} \frac{\mathrm{d} t}{t}=-k \ln (F(r) / q)
$$

from which

$$
x=F(r)=q \exp (-r / k) \quad r \geq 0, \quad q>0,
$$

being $\left\{b_{j}\right\}=\{k, q\}$ an additional set of new parameters. In consequence, from Equation (3), $W(r)$ is given by

$$
W(r)=\frac{1}{2 k}\left(1-\frac{c-(a+b+1) q \exp (-r / k)}{1-q \exp (-r / k)}\right)
$$

leading, from Equation (11), to

$$
\begin{aligned}
& W^{2}(r)+W^{\prime}(r)-g(F(r)) R(F(r)) \\
& =-\frac{q((a+b-c+1) c-2 a b) \exp (-r / k)}{2 k^{2}(1-q \exp (-r / k))^{2}}-\frac{q^{2}\left((c-1)^{2}-(a-b)^{2}\right) \exp (-2 r / k)}{4 k^{2}(1-q \exp (-r / k))^{2}}+\frac{(c-1)^{2}}{4 k^{2}} .
\end{aligned}
$$

Thus, by defining

$$
A+B=\frac{2 a b-c(a+b+1-c)}{2 k^{2}}
$$

and

$$
A-C=\frac{(c-1)^{2}-(a-b)^{2}}{4 k^{2}},
$$


it becomes possible to identify the potential

$$
V(r)=\frac{q A \exp (-r / k)}{1-q \exp (-r / k)}+\frac{q B \exp (-r / k)}{(1-q \exp (-r / k))^{2}}+\frac{q^{2} C \exp (-2 r / k)}{(1-q \exp (-r / k))^{2}},
$$

the eigenvalue

$$
E=-\left(\frac{c-1}{2 k}\right)^{2}
$$

and, from Equation (7), the eigenfunction

$$
\psi(r)=(q \exp (-r / k))^{(c-1) / 2}(1-q \exp (-r / k))_{2}^{(a+b-c+1) / 2}{ }_{2}(a, b ; c ; q \exp (-r / k)) .
$$

In what follow, the parameters $a, b$ and $c$ will be calculated by considering the boundary conditions of the system in order to have physically acceptable wavefunctions. For example, by combining Equation (89) and Equation (90) one obtains

$$
c=a+b-h
$$

with

$$
h=\left(1+4 k^{2}(B+C)\right)^{1 / 2} .
$$

Thus, to have a node in $r_{s}$ is necessary to apply the condition $\psi\left(r_{s}\right)=0$ in Equation (93) with $r_{s}=k \ln q$, the singular point of $V(r)$, which is achieved if

$$
a+b+1-c>0
$$

and

$$
B+C \geq 0 .
$$

On the other hand, by using Equation (94) in Equation (89) we obtain

$$
b=\frac{(h+1)(h-a)-2 k^{2}(A+B)}{h+1-2 a}
$$

and

$$
c=\frac{2 a(h-a)-2 k^{2}(A+B)}{h+1-2 a} .
$$

Also, since the hypergeometric function of Equation (93) is an infinite series, the condition

$$
a=-n, \quad n=0,1,2,3, \cdots
$$

leads to a polynomial of $n$ degree in the variable $q \exp (-r / k)$. Besides, $\psi(r) \rightarrow 0$ when $r \rightarrow \infty$ indicates that $\frac{c-1}{2}>0$ in the first exponent of Equation (93) which gives the number of states for the system

$$
0 \leq n<k \sqrt{C-A}-\frac{h+1}{2} .
$$

These assumptions on the original parameters $\{a, b, c\}$ assure that boundary conditions are fulfilled, leading to a physically acceptable wavefunction for the Schrödinger equation under consideration. Additionally, the energy spectra for the potential $V(r)$ is obtained by using Equation (99) in Equation (92). That is

$$
E_{n}=-\frac{1}{k^{2}}\left(\frac{\left(n+\frac{h+1}{2}\right)^{2}-k^{2}(C-A)}{2\left(n+\frac{h+1}{2}\right)}\right)^{2}
$$


with the corresponding wavefunctions

$$
\psi_{n}(r)=(q \exp (-r / k))^{(c-1) / 2}(1-q \exp (-r / k))^{(h+1) / 2}{ }_{2} F_{1}(-n, b ; c ; q \exp (-r / k)) .
$$

It should be pointed out that in this case the potential $V(r)$ has a minimum value

$$
V\left(r_{\min }\right)=-\frac{(A+B)^{2}}{4(B+C)}
$$

in

$$
r_{\min }=k \ln \left(\frac{q(A-B-2 C)}{A+B}\right)
$$

on condition that

$$
A+B<0 \leq B+C,
$$

since in this case the argument of the logarithm function, given in Equation (105), is always positive. Consequently, Equations (104)-(106) ensures that potential $V(r)$ is attractive with a infinite wall in $r_{s}$. In short, the potential given in Equation (91) is general and contains as particular cases exactly-solvable potentials for specific values of $A, B$, and $C$. For example, the choice of $A=\frac{\hbar^{2}}{2 m} V o, \quad B=C=0$ and $q=1$ lead to the exactly-solvable potential

$$
V(r)=\left(\frac{A \exp (-r / k)}{1-q \exp (-r / k)}\right)=\frac{\hbar^{2}}{2 m}\left(\frac{V o}{\exp (r / k)-1}\right)
$$

which is identified with the standard Hulthén potential with eigenvalues [15] [16]

$$
E_{n}=-\frac{\hbar^{2}}{2 m k^{2}}\left(\frac{(1+n)^{2}+k^{2} A}{2(n+1)}\right)^{2}
$$

and eigenfunctions

$$
\psi_{n}(r)=(\exp (-r / k))^{(c-1) / 2}(1-\exp (-r / k))_{2} F_{1}(-n, b ; c ; q \exp (-r / k)) .
$$

In a similar way, the selection $C=0$ and $A+B<0$ permit us to obtain the supersymmetric or generalized Hulthén potential already given by Morales et al., [17]. Also, the selection $A=V_{0}, B=0, C=\frac{\alpha(\alpha-1)}{b^{2}}$, $k=b$ and $q=1$ gives rise to the Manning-Rosen potential [18]

$$
V(r)=\frac{1}{b^{2}}\left(\frac{\alpha(\alpha-1) \exp (-2 r / b)}{(1-\exp (-r / b))^{2}}-\frac{A \exp (-r / b)}{1-\exp (-r / b)}\right)
$$

with energy spectra

$$
E_{n}=-\frac{1}{b^{2}}\left(\frac{A-\alpha}{2(\alpha+n)}-\frac{n(n+2 \alpha)}{2(\alpha+n)}\right)^{2}
$$

and wavefunctions

$$
\psi_{n}(r)=(1-\exp (-r / k))^{\alpha} \exp (-r / k)^{\frac{c-1}{2}}{ }_{2} F_{1}(-n, b ; c ; \exp (-r / b)) .
$$

Another important exactly-solvable exponential potential that have hypergeometric wavefunctions is the Woods-Saxon potential which has been worked using the Numerov method for the standard model [19] or by means of the Nikiforov-Uvarov procedure for the generalized case [20]. According to our results, one can show 
that the generalized Woods-Saxon potential is obtained as particular case of our proposal when choosing $A=V_{0}, \quad B=V_{1}, \quad C=0$ and $q=-\exp (2 a R)$, where $k=1 /(2 a)$, namely [21],

$$
V(r)=\frac{-V_{0}}{1+\exp (2 a(r-R))}-\frac{V_{1} \exp (2 a(r-R))}{[1+\exp (2 a(r-R))]^{2}} .
$$

However, due to the fact that the approach considered until now in this work is consistent with $q>0$, in the case $q<0$ some conditions have to be considered affecting wavefunctions and energy spectra as well as coordinates. That is, for $q$ negative, it must be necessary to change $q \rightarrow-q$ in the transformation $F(r)$, which means that $r$ is no longer the radial variable. Also, the conditions on the original parameters $a, b$ and $c$, given in Eqs.(94-99), will be corrected to $\psi( \pm \infty) \rightarrow 0$, that is,

$$
c=a+b+h,
$$

with

$$
h=\left(1+4 k^{2}(C-B)\right)^{1 / 2},
$$

and

$$
a+b<0<c-1,
$$

as well as

$$
C-B \geq 0 \text {. }
$$

Thus, by using Equation (114) and the negative of Equation (89) we obtain

$$
b=\frac{(1-h)(h+a)-2 k^{2}(A+B)}{h-1+2 a},
$$

and

$$
c=\frac{2 a(h+a)-2 k^{2}(A+B)}{h-1+2 a},
$$

with the condition $a=-n$, for $n=0,1,2,3, \cdots$. Thus, with these new parameters, the energy spectra and wavefunctions of the generalized Wood-Saxon potential are respectively

$$
E_{n}=-\frac{1}{k^{2}}\left(\frac{\left(n+\frac{1-h}{2}\right)^{2}-k^{2}(C+A)}{2\left(n+\frac{1-h}{2}\right)}\right)^{2},
$$

and

$$
\psi_{n}(r)=\left(\exp \left(-\left(r-R_{0}\right) / 2\right)\right)^{(c-1) / 2}\left(1+\exp \left(-\left(r-R_{0}\right) / a\right)\right)^{\frac{1-h}{2}}{ }_{2} F_{1}\left(a, b ; c ;-\exp \left(-\left(r-R_{0}\right) / 2\right)\right) .
$$

b). Option with $g(x) \sim \frac{P(x)}{x^{2}}$.

Similarly to the above case, in this new situation one have

$$
g(x)=\frac{1-x}{k^{2} x} \quad k>0 \text { and } x \neq 0,
$$

that leads to the transformation

$$
x=F(r)=1-\gamma \exp (-r / k) \quad r \geq 0
$$

where $\gamma=(1-q)$, and 


$$
W(r)=\frac{1}{2 k}\left(\frac{c}{1-\gamma \exp (-r / k)}-(a+b)\right)
$$

that, from Equation (3), lets write

$$
\begin{aligned}
& W^{2}(r)+W^{\prime}(r)-g(F(r)) R(F(r)) \\
& =\frac{1}{4 k^{2}}\left(\frac{c^{2}-2 c(a+b)+(2 a b+c(a+b-1)) 2 \gamma \exp (-r / k)}{(1-\gamma \exp (-r / k))^{2}}\right) \\
& -\frac{1}{4 k^{2}}\left(\frac{4 a b \gamma^{2} \exp (-2 r / k)}{(1-\gamma \exp (-r / k))^{2}}-(a+b)^{2}\right) .
\end{aligned}
$$

In this new situation, by considering the identities

$$
A+B=\frac{4 a b+c(a+b)}{4 k^{2}}
$$

and

$$
A-C=\frac{a b}{4 k^{2}}
$$

as well as

$$
F=\frac{c^{2}-2 c(a+b)}{4 k^{2}}
$$

one find the multiparameter exponential-type potential

$$
V(r)=\frac{\gamma A \exp (-r / k)}{1-\gamma \exp (-r / k)}+\frac{\gamma B \exp (-r / k)}{(1-\gamma \exp (-r / k))^{2}}+\frac{\gamma^{2} C \exp (-2 r / k)}{(1-\gamma \exp (-r / k))^{2}}+\frac{F}{(1-\gamma \exp (-r / k))^{2}},
$$

with eigenvalue

$$
E=-\left(\frac{a+b}{2 k}\right)^{2}
$$

and eigenfunction

$$
\psi(r)=(\gamma \exp (-r / k))^{(a+b-c) / 2}(1-\gamma \exp (-r / k))^{c / 2}{ }_{2} F_{1}(a, b ; c ; 1-\gamma \exp (-r / k)) .
$$

At first glance, the potential of Equation (129) can be considered as a new one and however it should be noticed that it can be written as

$$
\mathrm{V}(r)=V(r)-F=\frac{\gamma A \exp (-r / k)}{1-\gamma \exp (-r / k)}+\frac{(B+2 F) \gamma \exp (-r / k)}{(1-\gamma \exp (-r / k))^{2}}+\frac{(C-F) \gamma^{2} \exp (-2 r / k)}{(1-\gamma \exp (-r / k))^{2}}
$$

That is, the above $\mathrm{V}(r)$ has the same structure of potential given in Equation (91) which means that leads to similar results to those shown in Section 3.3.a). Also, thinking in molecular physics applications, the usefulness of the proposed exactly solvable multiparameter exponential-type potential given in Equation (91) is focused to obtain vibrational modes of diatomic molecules. At this regard, for the study of bound states solutions, one option is a redefinition of parameter $B$ in order to include the centrifugal term and then to apply some of the approximation schemes proposed to $1 / r^{2}$. For example, one can consider $q=1$ and $B=\ell(\ell+1) / k^{2}$ with $\exp (-r / k) /(1-\exp (-r / k))^{2} \approx 1 / r^{2}$ and so on; see for example Falaye et al. [22]. Moreover, in the same way, other particular radial potentials can be derived from our proposal as, for example, the recently considered Man- 
ning-Rosen potential [23] which, as explained before, comes from Equation (91).

\section{Concluding Remarks}

In this paper, we present a method to obtain the general canonical form of second order differential equations on the field of theoretical physics. The procedure is similar to the method proposed by Levai [24] and however we consider a general DE to be converted into a Schrödinger-like equation. The algorithm we propose, makes use of an auxiliary function $g(x)$ to find the needed transformation to obtain the exactly-solvable potentials with the same wavefunctions of the original DE. To show the usefulness of the proposed method, their application to the hypergeometric DE along with different forms of $g(x)$ is given explicitly. Thus, for each $g(x)$ we found a generalized exactly-solvable potential from where well known potential models are obtained as particular cases. For example, the choice $g(x)=$ const gives place to the trigonometric and hyperbolic Scarf potentials as well as to the Posh-Teller potential, while the choice of $g(x)$ proportional to $P(x)$ leads to the Eckart and to the trigonometric and hyperbolic Rosen-Morse potentials. Similarly, with the choice of $g(x) \sim x^{2} / P(x)$ we obtain a generalized exactly-solvable multiparameter ( $A, B, C, q$ and $k$ ) exponential-type potential that contains, depending on the parameters selection, the particular cases of the Hulthén, Manning-Rosen and Wood-Saxon potentials, standard and generalized, among others. Besides, the proposed multiparameter exponential-type potential can be used in the search of bound state solutions of specific potential models of diatomic molecules in the frame of different approaches to the centrifual term [25]. In short, with the application given in this work we have shown that it is possible to give a unified treatment of exactly-solvable potentials having hypergeometric wavefunctions as common feature. Finally, we want to notice that the method is general for which can be applied to other DE as for example on the position-dependent mass Schrödinger equation. Furthermore, advantageously, our proposal avoids the use of specialized solution methods applied to particular potential models as has been usually done.

\section{Acknowledgements}

This work was partially supported by the projects UAM-A-CBI-2232004 and 009. JGR thanks to the Instituto Politécnico Nacional for the financial support given through the COFAA-IPN project SIP-200150019. JGM acknowledges to the IPN-ESFM, for the hospitality during his PhD studies in Science and Technology.

\section{References}

[1] Sukumar, C.V. (1985) Supersymmetric Quantum Mechanics of One-Dimensional Systems. Journal of Physics A: Mathematical and General, 18, 2917-2936. http://dx.doi.org/10.1088/0305-4470/18/15/020

[2] De, R., Dutt, R. and Sukhatme, U. (1992) Mapping of Shape Invariant Potentials under Point Canonical Transformations. Journal of Physics A: Mathematical and General, 25, L843-L850. http://iopscience.iop.org/0305-4470/25/13/013

[3] Setare, M.R. and Karimi, E. (2008) Mapping of Shape Invariant Potentials by the Point Canonical Transformation. International Journal of Theoretical Physics, 47, 891-897.

[4] Cooper, F., Khare, A. and Sukhatme, U. (1995) Supersymmetry and Quantum Mechanics. Physics Reports, $251267-$ 385. http://dx.doi.org/10.1016/0370-1573(94)00080-M

[5] Ho, C.-L. (2009) Simple Unified Derivation and Solution of Coulomb, Eckart and Rosen-Morse Potentials in Prepotential Approach. Annals of Physics, 324, 1095-1104. http://dx.doi.org/10.1016/j.aop.2008.10.004

[6] Jia, C.-S., Diao, Y.-F., Li, M., Yang, Q.-B., Sun, L.-T. and Huang, R.-Y. (2004) Mapping of the Five-Parameter Exponential-Type Potential Model into Trigonometric-Type Potentials. Journal of Physics A: Mathematical and General, 37 11275-11284. http://dx.doi.org/10.1088/0305-4470/37/46/012

[7] Jia, C.-S., Yi, L.-Z., Zhao, X.-Q., Liu, J.-Y. and Sun, L.-T. (2005) Systematic Study of Exactly Solvable Trigonometric Potentials with Symmetry. Modern Physics Letters A, 20, 1753-1762. http://dx.doi.org/10.1142/S0217732305017081

[8] Jia, C.-S., Liu, J.-Y., Sun, Y., He, S. and Sun, L.-T. (2006) A Unified Treatment of Exactly Solvable Trigonometric Potential Models. Physica Scripta, 73, 164-168. http://iopscience.iop.org/1402-4896/73/2/006

[9] Cooper, F., Khare A. and Sukhatme, U. (2001) Supersymmetry in Quantum Mechanics. World Scientific Publishing Co Pte Ltd.

[10] Ciftci, H., Hall, R.L. and Saad, N. (2003) Asymptotic Iteration Method for Eigenvalue Problems. Journal of Physics A: Mathematical and General, 36, 11807-11816. http://dx.doi.org/10.1088/0305-4470/36/47/008 
[11] Nikiforov, A.F. and Uvarov, V.B. (1988) Special Functions of Mathematical Physics. Birkhauser, Basel. http://dx.doi.org/10.1007/978-1-4757-1595-8

[12] Peña, J.J., Morales, J., García-Martínez, J. and García-Ravelo, J. (2008) Exactly Solvable Quantum Potentials with Special Functions Solutions. International Journal of Quantum Chemistry, 108, 1750-1757. http://dx.doi.org/10.1002/qua.21611

[13] Abramowitz, M. and Stegun, I.A. (1972) Handbook of Mathematical Functions. Wiley and Sons, New York.

[14] Granville, W.A., Smith, P.F. and Longley, W.R. (1941) Elements of the Differential and Integral Calculus. Ginn \& Co., Boston.

[15] Hulthén, L. (1942) On the Characteristic Solutions of the Schrödinger Deuteron Equation. Arkiv för Matematik Astronomi och Fysik A, 28, art 5: 1-12.

[16] Chen, G. (2004) Shape Invariance and the Supersymmetric WKB Approximation for the Generalized Hulthén Potential. Physica Scripta, 69, 257-259. http://dx.doi.org/10.1238/physica.regular.069a00257

[17] Morales, J., Peña, J.J. and Morales-Guzman, J.D. (2000) The Generalized Hulthén Potential. Theoretical Chemistry Accounts, 104, 179-182. http://dx.doi.org/10.1007/s002140000130

[18] Ahmed, S.A.S. and Buragohain, L. (2010) Exactly Solved Potentials Generated from the Manning-Rosen Potential Using Extended Transformation Method. Electronic Journal of Theoretical Physics, 7, 145-154.

[19] Fatah, A.H. (2012) Calculation of the Eigenvalues for Wood-Saxon's Potential by Using Numerov Method. Advances in Theoretical and Applied Mechanics, 5, 23-31.

[20] Berkdemir, C., Berkdemir, A. and Sever, R. (2005) Polynomial Solutions of the Schrödinger Equation for the Generalized Woods-Saxon Potential. Physical Review C, 72, 027001-1-027001-4.

[21] Gönül, B. and Köksal, K. (2007) Solutions for a Generalized Woods-Saxon Potential. Physica Scripta, 76, 565-570. http://dx.doi.org/10.1088/0031-8949/76/5/026

[22] Falaye, B.J., Oyewumi, K.J., Ibrahim, T.T., Punyasena, M.A. and Onate, C.A. (2013) Bound State Solutions of the Manning-Rosen Potential. Canadian Journal of Physics, 91, 98-104. http://dx.doi.org/10.1139/cjp-2012-0330

[23] Nasser, I., Abdelmonem, M.S. and Abdel-Hady, A. (2013) The Manning-Rosen Potentials Using J-Matrix Approach. Molecular Physics, 111, 1-8. http://dx.doi.org/10.1080/00268976.2012.698026

[24] Lévai, G. (1989) A Search for Shape-Invariant Solvable Potentials. Journal of Physics A: Mathematical and General, 22, 689-702. http://dx.doi.org/10.1088/0305-4470/22/6/020

[25] Peña, J.J., García-Martínez, J., García-Ravelo, J. and Morales, J. (2014) l-State Solutions of Multiparameter Exponential-Type Potentials. Journal of Physics: Conference Series, 490, 012199. http://dx.doi.org/10.1088/1742-6596/490/1/012199

[26] Arfken, G. and Weber, H. (2005) Mathematical Methods for Physicists. 6th Edition, Elsevier AP, Boston.

[27] Polyani, A.D. and Zaistev, V.F. (2003) Handbook of Exact Solutions for Ordinary Differential Equations. 2nd Edition, Chapman\& Hall/CRC, Boca Raton, New York. 


\section{Appendix}

The general second order DE

$$
P(x) f^{\prime \prime}(x)+Q(x) f^{\prime}(x)+R(x) f(x)=0,
$$

is transformed into their standard canonical form [26]

$$
v^{\prime \prime}(x)+S(x) v(x)=0
$$

by means of the transformation

$$
f(x)=\exp \left(-\frac{1}{2} \int \frac{Q(x)}{P(x)} \mathrm{d} x\right) v(x) .
$$

i.e.

$$
v^{\prime \prime}(x)-\left[\frac{1}{4}\left(\frac{Q(x)}{P(x)}\right)^{2}+\frac{1}{2} \frac{\mathrm{d}}{\mathrm{d} x}\left(\frac{Q(x)}{P(x)}\right)-\frac{R(x)}{P(x)}\right] v(x)=0,
$$

where apostrophes indicates derivative with respect to the argument.

In order to have a generalized canonical transformation of Equation A1, we consider the variable change

$$
x=F(r)
$$

to obtain the general transformation [27]

$$
f(F(r))=\sqrt{F^{\prime}(r)} \exp \left(-\frac{1}{2} \int \frac{Q(F(r))}{P(F(r))} \mathrm{d} F(r)\right) \psi(r) .
$$

Consequently, one have

$$
\psi^{\prime \prime}(r)+S(r) \psi(r)=0
$$

being

$$
S(r)=-\mathrm{W}^{2}(r)-\mathrm{W}^{\prime}(r)+\frac{R(F(r))}{P(F(r))}\left(F^{\prime}(r)\right)^{2},
$$

where

$$
\mathrm{W}(r)=\frac{1}{2}\left(\frac{Q(F(r)) F^{\prime}(r)}{P(F(r))}-\frac{F^{\prime \prime}(r)}{F^{\prime}(r)}\right) .
$$

It will be noticed that, for the particular case $x=F(r)=r$ Equation (A8) reduces to the expression given in the square parenthesis of Equation (A4). 